\title{
Is perinatal asphyxia predictable?
}

\author{
Anna Locatelli ${ }^{i *}$, Laura Lambicchi ${ }^{2}$, Maddalena Incerti ${ }^{2}$, Francesca Bonati ${ }^{1}$, Massimo Ferdico ${ }^{3}$, Silvia Malguzzi ${ }^{4}$,
} Ferruccio Torcasio ${ }^{5}$, Patrizia Calzi ${ }^{6}$, Tiziana Varisco ${ }^{7}$ and Giuseppe Paterlini ${ }^{4}$

\begin{abstract}
Background: The objective of our study was to evaluate the association between perinatal asphyxia and hypoxicischemic encephalopathy (HIE) with the presence of ante and intrapartum risk factors and/or abnormal fetal heart rate (FHR) findings, in order to improve maternal and neonatal management.
\end{abstract}

Methods: We did a prospective observational cohort study from a network of four hospitals (one Hub center with neonatal intensive care unit and three level I Spoke centers) between 2014 and 2016. Neonates of gestational age $\geq 35$ weeks, birthweight $\geq 1800 \mathrm{~g}$, without lethal malformations were included if diagnosed with perinatal asphyxia, defined as $\mathrm{pH} \leq 7.0$ or Base Excess (BE) $\leq-12 \mathrm{mMol} / \mathrm{L}$ in Umbical Artery (UA) or within $1 \mathrm{~h}, 10 \mathrm{~min}$ Apgar $<5$, or need for resuscitation $>10$ min.

FHR monitoring was classified in three categories according to the American College of Obstetricians and Gynecologists (ACOG). Pregnancies were divided into four classes: 1) low risk; 2) antepartum risk; 3) intrapartum risk; 4) and both ante and intrapartum risk.

In the first six hours of life asphyxiated neonates were evaluated using the Thomson score (TS): if TS $\geq 5$ neonates were transferred to Hub for further assessment; if TS $\geq 7$ hypothermia was indicated.

Results: Perinatal asphyxia occurred in $21.5 \%$ cases $(321 / 14,896)$ and HIE in $1.1 \%$ o $(16 / 14,896)$. The total study population was composed of 281 asphyxiated neonates: 68/5152 (1.3\%) born at Hub and 213/9744 (2.2\%) at Spokes $(p<0.001$, OR 0.59, 95\% Cl 0.45-0.79). 32/213 (15\%) neonates were transferred from Spokes to Hub. Overall, 12/281 were treated with hypothermia. HIE occurred in 16/281 (5.7\%) neonates: four grade I, eight grade II and four grade III. Incidence of HIE was not different between Hub and Spokes.

Pregnancies resulting in asphyxiated neonates were classified as class 1) $1.1 \%$, 2) $52.3 \%$, 3) $3.2 \%$, and 4) $43.4 \%$. Sentinel events occurred in $23.5 \%$ of the cases and FHR was category II or III in $50.5 \%$ of the cases. $40.2 \%$ cases of asphyxia and $18.8 \%$ cases of HIE were not preceded by sentinel events or abnormal FHR.

Conclusions: We identified at least one risk factor associated with all cases of HIE and with most cases of perinatal asphyxia. In absence of risk factors, the probability of developing perinatal asphyxia resulted extremely low. FHR monitoring alone is not a reliable tool for detecting the probability of eventual asphyxia.

Keywords: Hypoxic-ischemic encephalopathy; asphyxia; sentinel events, Nulliparity, Umbilical artery pH, Fetal heart rate monitoring

\footnotetext{
* Correspondence: anna.locatelli@unimib.it

'Department of Obstetrics and Gynecology, ASST Vimercate, Carate B.za

Hospital, University of Milano-Bicocca, Monza, Italy

Full list of author information is available at the end of the article
}

(c) The Author(s). 2020 Open Access This article is licensed under a Creative Commons Attribution 4.0 International License, which permits use, sharing, adaptation, distribution and reproduction in any medium or format, as long as you give appropriate credit to the original author(s) and the source, provide a link to the Creative Commons licence, and indicate if changes were made. The images or other third party material in this article are included in the article's Creative Commons licence, unless indicated otherwise in a credit line to the material. If material is not included in the article's Creative Commons licence and your intended use is not permitted by statutory regulation or exceeds the permitted use, you will need to obtain permission directly from the copyright holder. To view a copy of this licence, visit http://creativecommons.org/licenses/by/4.0/ The Creative Commons Public Domain Dedication waiver (http://creativecommons.org/publicdomain/zero/1.0/) applies to the data made available in this article, unless otherwise stated in a credit line to the data. 


\section{Background}

Cases of neonatal encephalopathy related to perinatal asphyxia are called hypoxic-ischemic encephalopathy (HIE) and can lead to long-term neurological sequelae and cerebral palsy $[1,2]$.

The Sarnat and Sarnat classification [3] is still the universally accepted scoring system to provide information about the prognosis for the asphyxiated neonate. This staging is based on the infant's clinical presentation, examination findings and the presence of seizures, with emphasis on the duration of symptoms.

Both clinical (Thompson scale [4]) and instrumental (cerebral function monitor/aEEG) assessment are validated by international literature for classifying the severity of damage immediately after birth. This allows for initiating the correct treatment (ie Therapeutic Hypothermia) as soon as possible.

Perinatal asphyxia is defined as at least one of the following characteristics in a neonate: $10 \mathrm{~min}$ Apgar score $\leq$ 5 , need for resuscitation $>10 \mathrm{~min}$, metabolic acidosis ( $\mathrm{pH} \leq 7.0$ or $\mathrm{BE} \leq-12 \mathrm{mMol} / \mathrm{L}$ in umbilical artery (UA) or within $1 \mathrm{~h}$ of life).

There is a dose-dependent relationship between the degree of acidosis and the likelihood of adverse neonatal outcome $[5,6]$.

Both ante and intrapartum risk factors have been associated with perinatal asphyxia and HIE. Some of these are clinically recognized as asphyxial birth events (sentinel events): uterine rupture, placental abruptio, shoulder dystocia and cord prolapse. However, the majority of risk factors (preeclampsia, intrauterine growth restriction, intrapartum fever, emergency delivery) seem to interact in the causal pathway to HIE rather than being its direct antecedent [7-11]. In addition to the historically described risk factors, new ones have recently been identified, such as obesity and previous cesarean section [12]. Nevertheless, results from a large registry of neonatal encephalopathy show that half of the neonates had neither asphyxia nor inflammatory indicators, suggesting that a large number of cases remain unexplained [13].

Fetal heart rate (FHR) monitoring provides important information about fetal status. However, its role in the prediction of fetal asphyxia remains controversial, despite efforts directed at its interpretation and the development of protocols for the management of abnormal patterns $[14,15]$.

To summarize, there are a number of conditions that have been associated with the presence of perinatal asphyxia and its extreme consequence, HIE. Nevertheless, it is problematic to identify the sequence of events that leads to perinatal asphyxia during prenatal and intrapartum care.

In this context the objective of our study was to evaluate the association between perinatal asphyxia and HIE with the presence of risk factors and/or abnormal FHR findings in order to improve maternal and neonatal management.

\section{Methods}

We conducted a prospective observational cohort study between July 2014 and June 2016 from a network of four hospitals with different levels of care which shared protocols of intrapartum and neonatal care.

Inclusion criteria were asphyxiated neonates of gestational age $\geq 35$ weeks, birthweight $\geq 1800 \mathrm{~g}$, and no severe malformations. Perinatal asphyxia was defined as $\mathrm{pH}$ $\leq 7.0$ or $\mathrm{BE} \leq-12 \mathrm{mMol} / \mathrm{L}$ in UA or within $1 \mathrm{~h}, 10 \mathrm{~min}$ Apgar $\leq 5$, or need for resuscitation $>10 \mathrm{~min}$ [16].

The network includes one Hub University Hospital center with a neonatal intensive care unit that offers therapeutic hypothermia, and three level I Community Hospital Spoke centers.

In Spoke centers, only women with low risk pregnancies or minor complications can be admitted for delivery. High risk pregnancies for medical or fetal complications are referred to Hub. Patients with low risk pregnancies may choose to deliver in either Spoke or Hub centers.

Perinatal mortality rate in our network is superimposable to the general Italian one, that is $4 \%$.

The Italian childbirth system offers universal and free of charge maternity care.

The acid-base status is routinely determined at birth by UA and, if not feasible, within one hour of life on the neonate.

Data regarding maternal history, pregnancy complications, labor and delivery were collected.

The uterine contraction changes during labor were monitored and prospectively registered. Tachysystole is defined as $\geq 5$ contractions in $10 \mathrm{~min}$ for $30 \mathrm{~min}$ or more.

Fetal electronic monitoring on admission and/or during labor was prospectively collected and managed using the Clark algorithm [17]. The FHR was monitored intermittently during the first and second stages of labor, at least every $15 \mathrm{~min}$ for $1 \mathrm{~min}$ in the active phase of the first stage of labor and after every contraction in the second stage. Continuous electronic monitoring was implemented in the presence of obstetric or medical risk factors evaluated on admission and during labor, or if the FHR was abnormal at intermittent auscultation. The last hour tracing was reviewed by two authors (FB, MI), blinded to the outcome of the neonate, and it was classified in 3 categories according to ACOG classification. Under this classification system, category I tracings were defined as including all of the following characteristics: baseline rate between 110 and 160 beats per minute, moderate variability, and absence of late or variable decelerations. Category III tracings were defined as absent variability with recurrent late decelerations, recurrent 
variable decelerations, or bradycardia or sinusoidal pattern. Category II tracings include those patterns that did not meet the criteria of categories I or III. Discrepancies in category status of tracing were adjudicated by an additional reviewer (AL) blinded to the assessments of the initial reviewers.

We divided pregnancies into four classes: low risk (no risk factors); antepartum risk (nulliparous women, maternal age $>40$ years, $\mathrm{BMI}>30$, oligohydramnios or polyhydramnios, fetal growth restriction, induction of labor, maternal disease, previous cesarean); intrapartum risk (sentinel event, thick meconium during labor, fever, tachysystole); and both ante and intrapartum risk.

Data recorded on each infant included: $\mathrm{pH}, \mathrm{BE}$, lactate, need for ventilation by mask or endotracheal tube during initial stabilization, need for cardiac massage, biochemical and instrumental data. In the first six hours of life, asphyxiated neonates were monitored with the Thomson score [4]: if TS was $\geq 5$, the neonate was transferred to the Hub for further surveillance; if TS was $\geq 7$ hypothermia was indicated.

Encephalopathy was classified according to Sarnat \& Sarnat [3].

Neurodevelopmental follow-up was offered to all neonates until 1 year of age and to neonates with moderate or severe encephalopathy until 6 years of age.

Written informed consent was obtained from all participants.

Descriptive statistics were calculated as mean (standard deviation) for numerical variables and absolute frequencies and percentages for categorical variables. Statistical analysis included univariate analyses with Student T-test, one-way ANOVA, chi-square and Fisher exact test. Multivariate logistic regression analyses were performed to control for confounding variables (SPSS). A two-tailed $p$ value $<.05$ was considered significant. SPSS (IBM Corp. version 24) was used to run the analyses.

\section{Results}

During the study period perinatal asphyxia occurred in $321 / 14,896$ cases $(21.5 \%$ ) and HIE in $16 / 14,896$ (1.1\%o).

Asphyxia occurred in 1.5\% (79/5152) in the Hub center, in $1.8 \%(64 / 3552)$ in Spoke 1, 2.9\% (94/3269) in Spoke 2 and $2.9 \%$ in Spoke 3 (84/2923).

We excluded 40 neonates because they did not meet the inclusion criteria (two cases of neonatal diagnosis of metabolic disease), or because we did not have parental consent, or we had incomplete data for enrolment. Thus, 281 asphyxiated neonates were included in the analysis: 68/5152 (1.3\%) delivered at the Hub and 213/9744 $(2.2 \%)$ in Spoke centers $(p<0.001$, OR $0.59,95 \%$ CI $0.45-0.79)$.

Of the 281 asphyxiated neonates, $80 / 281$ had UA pH $\leq$ 7 (28\%), 261/281 had BE $\leq-12$ (93\%) (of which 51
(18\%) with $\mathrm{BE} \leq-16) ; 189 / 281(67 \%)$ neonates were enrolled only for $\mathrm{BE} \leq-12$.

There were no cases of HIE among neonates who were not enrolled.

There were no perinatal deaths during the study period.

Descriptive characteristics of asphyxiated neonates are reported in Table 1.

During neonatal monitoring TS was $\geq 5$ in $24 / 281$ cases $(8.5 \%)$ and $32 / 213(15 \%)$ neonates were transferred from Spokes to the Hub Center.

Overall, 12/281 neonates received hypothermia. There were $9 / 281$ cases of neonatal seizures. HIE was diagnosed in $16 / 281(5.7 \%$,) including four with grade I, eight with grade II, and four with grade III HIE. Asphyxia and HIE according to place of birth are shown in Fig. 1. No differences in incidence of HIE were observed between Hub and Spokes: 7/5152 (0.13\%) vs 9/9744 $(0.09 \%), p=0.58, \mathrm{OR}=1.495 \%$ CI $0.44-49$. The detail of every case is reported in Table 2 .

Pregnancies were classified as low risk in $3 / 281(1.1 \%)$ cases, with antepartum risk in 147/281 (52.3\%), intrapartum risk in $9 / 281(3.2 \%)$, and both ante and intrapartum risk in 122/281 (43.4\%) cases (Fig. 2).

There were 214/281 (76.2\%) nulliparous women equally distributed in class 2 and 4, 16/281 (5.7\%) women $>40$ years, $87 / 281$ (31.0\%) with BMI $>30,14 / 281$ (5.0\%) cases of oligohydramnios, 20/281 (7.1\%) cases of polyhydramnios and $8 / 281(2.8 \%)$ cases of fetal growth restriction. We recorded 18/281 (6.4\%) cases of hypertensive disorders of pregnancy, 4/281 (1.4\%) positive thrombophilic status, $5 / 281(1.8 \%)$ pre-gestational and $31 / 281$ (11.0\%) gestational diabetes mellitus, 25/281 (8.9\%) thyroid diseases, $8 / 281(2.8 \%)$ gestational cholestasis, $1 / 281(0.4 \%)$ antepartum hemorrhage and 19/281 $(6.8 \%)$ previous cesarean section.

Table 1 Neonatal characteristics

\begin{tabular}{|c|c|c|}
\hline & N (\%) & Mean \pm SD (range) \\
\hline Male & $154(55)$ & \\
\hline Birth weight (g) & & $3261 \pm 435(4400-2020)$ \\
\hline $\mathrm{pH} \cup \mathrm{A}$ & & $7.05 \pm 0.11(7.34-6.66)$ \\
\hline $\mathrm{pH} \cup \mathrm{A} \leq 7$ & $80(28.5)$ & \\
\hline BE UA & & $-14.1 \pm 2.3(-5.3--22)$ \\
\hline $\mathrm{BE} \cup \mathrm{A} \leq-12$ & $261(92.8)$ & \\
\hline $\mathrm{BE} \cup \mathrm{A} \leq-16$ & $51(18.1)$ & \\
\hline Lactate UA (mmol/L) & & $10.5 \pm 2.8(18.4-3.7)$ \\
\hline 5 min Apgar $<5$ & $16(5.7)$ & \\
\hline 10 min Apgar < 5 & $5(1.8 \%)$ & \\
\hline Neonatal resuscitation $>10^{\prime}$ & $17(6)$ & \\
\hline
\end{tabular}

Legend: UA Umbilical Artery, BE Base Excess 


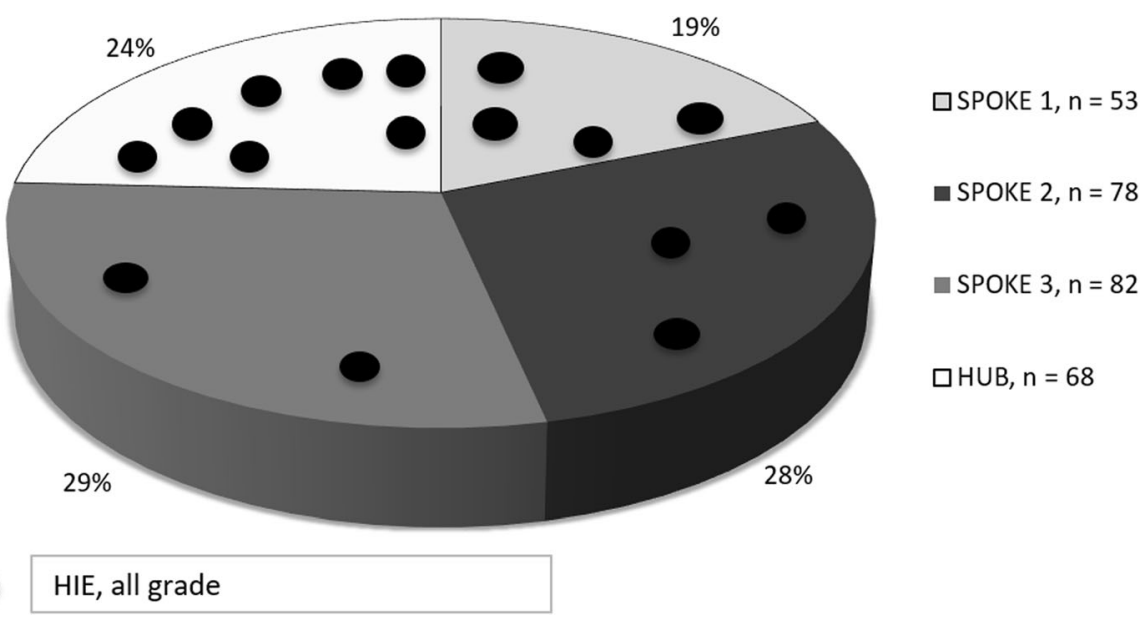

Fig. 1 Asphyxia and hypoxic-ischemic encephalopathy according to place of birth

Labor was induced in 96/281 (34.2\%) of cases. Thick meconium during labor was observed in 21/281 cases (7.5\%), fever or chorioamnionitis in $17 / 281$ (6.0\%) and tachysystole in 52/281 (18.5\%).

Sentinel events occurred in 66/281 cases (23.5\%) and preceded HIE in 6/16 (37.5\%), as described in detail in Fig. 3. Incidence of sentinel events was not different among neonates with HIE and others $(p=0.4089)$.

Only three cases of asphyxia (1\%) and no cases of HIE occurred in class 1 (low risk pregnancies). There were no cases of $\mathrm{UA} \mathrm{pH} \leq 7$ in this class.

There were $67 / 281$ (24\%) operative vaginal deliveries and 56/281 (20\%) cesarean sections including 39/56 (70\%) because of fetal distress. FHR category II and III in the last hour before delivery were documented in $142 / 281(50.5 \%)$ cases of perinatal asphyxia, and they preceded HIE in 9/16 (56.3\%) respectively. In 7/142 cases, FHR category II or III was observed before labor.

Moreover, FHR category II or III occurred in $61 / 80$ (76\%) cases with UA $\mathrm{pH} \leq 7$ compared with 81/201 (40\%) cases with UA pH $>7(p<0.001$ OR $4.895 \% \mathrm{CI}$ 2.6-8.9). HIE occurred more often if UA pH was $\leq 7$ (12/ $80,15.0 \%)$ in respect to case with UA $\mathrm{pH}>7$ (4/137, $3.0 \%)(p=0.002$, OR $5.995 \%$ CI 1.7-22.5).

$113 / 281(40.2 \%)$ cases of asphyxia and 3/16 (18.8\%) cases of HIE were not preceded by sentinel events or abnormal FHR findings.

\section{Discussion}

In our network the rates of neonatal asphyxia (1\%), as well as of $\mathrm{pH} \leq 7$ (5\%) and HIE (0.7-1.7\%o) were comparable to those described in literature when similar definitions are used $[5,6,18-22]$.
As expected, only a minority of asphyxiated neonates developed HIE (5.7\%) and they were promptly identified. Thanks to our shared care protocols and cooperative management system, appropriate care was guaranteed for all asphyxiated neonates, while minimizing the number of newborns transferred.

In our series the number of sentinel events in cases of perinatal asphyxia and HIE was as high as previously described in literature $(15-35 \%)$ [7, 13]. Emergency delivery can change the prognosis of these events with an appropriate diagnosis, management and team organization. Nevertheless, in some cases the clinical presentation reflects a chronic damage that has already occurred instead of an acute event (i.e. placental abruption), limiting the therapeutic options. In other cases, when a non-reassuring signal occurs (i.e. bradycardia), the available time to prevent fetal damage may be too short to alter the outcome (i.e. uterine rupture).

Many authors have focused on searching for risk factors associated with perinatal asphyxia and neonatal encephalopathy: a wide number of antecedents has been identified, nonetheless a variable percentage of the events remain unexplained $[8,9,11]$. In order to classify pregnancies into different classes of risk, we prospectively collected all known risk factors in our population, including the new emerging ones (eg. previous cesarean section, obesity). Low risk pregnancies were only a minor proportion $(1.1 \%)$ of our population and no cases of HIE presented in this group. This means that we managed to identify at least one risk factor (ante and/or intrapartum) associated with every case of HIE and of perinatal asphyxia, except 3 cases of perinatal asphyxia that did not have any identified risk factor. Therefore, we can assume that at least one of the risk factors we examined underlies the phenomenon of perinatal asphyxia 


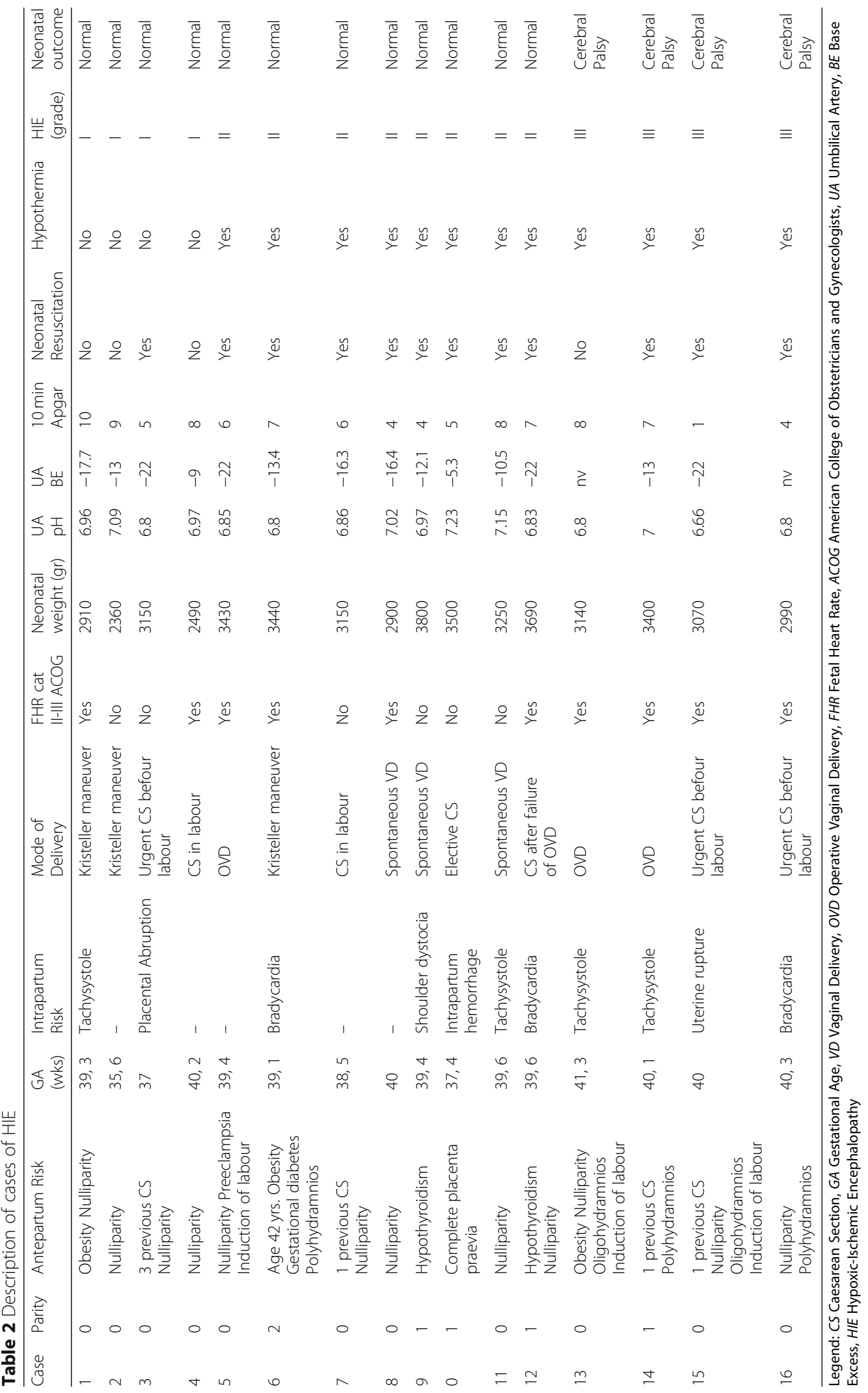




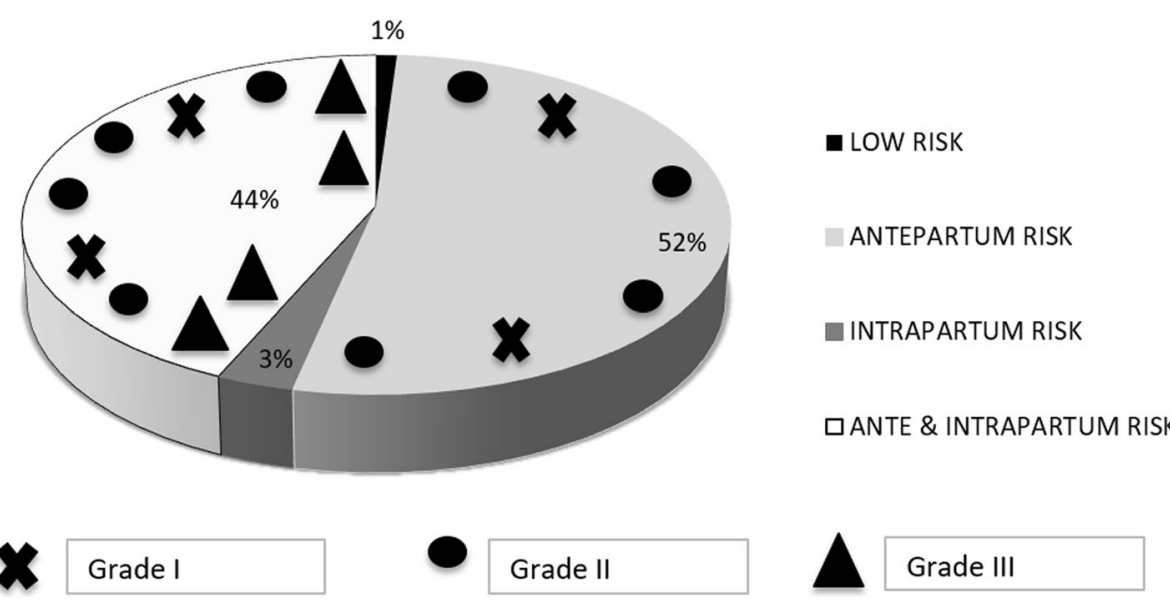

Fig. 2 Cases of hypoxic-ischemic encephalopathy and grade in asphyxiated neonates according to presence of risk factors

even if we cannot always identify a causal relation. More importantly, the probability of developing perinatal asphyxia in this low-risk population appears to be extremely low.

Our study design does not allow us to assign a relative risk or hazard ratio to the risk factors recorded.

The most common risk factor for asphyxia is nulliparity. It is identified in $76 \%$ of cases $(51 \%$ in our general population) and it is the only risk factor in $16 \%$ of cases. This is obviously a non-modifiable condition and it is widespread in the general obstetric population. Nonetheless, it has been previously described in association with need for cooling [21, 22]. The role of nulliparity could be explained by the increased frequency of pregnancy complications related to it, such as defective placentation and impaired fetal growth at term, undetected by routine clinical exams. Moreover, because of the longer duration of labor, nulliparas are at greater risk of infection and fetal intolerance to labor, interventions, operative deliveries, and associated perinatal events.

In spite of this, a recent study of Liljestrom [23] reported that in HIE cases an obstetric emergency occurred more commonly in the parous than in the nulliparous women, suggesting that intrapartum complications can occur also in women at lower risk, representing a major challenge in obstetrics.

The second most frequent risk factor is fetal tachysystole, which presented in 52/281 cases (18.5\%). In most

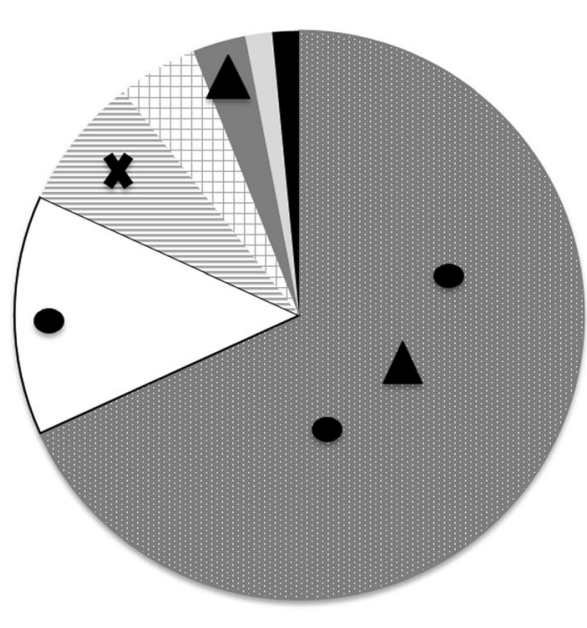

fetal bradycardia, $n=45$

$\square$ shoulder dystocia, $\mathrm{n}=9$

placental abruption, $n=5$

cord prolapse, $\mathrm{n}=3$

uterine rupture, $\mathrm{n}=2$

vasa previa rupture, $\mathrm{n}=1$

amniotic fluid embolism, $\mathrm{n}=1$

Grade I

Grade II

Grade III

Fig. 3 Cases of hypoxic-ischemic encephalopathy and grade in asphyxiated neonates according to presence of sentinel events 
cases, tachysystole was associated with other risk factors, such as the need for induction of labor (23 cases) and/or oxytocin augmentation (29 cases). Use of oxytocin can lead to excessive uterine activity, which has been associated with neonatal morbidity both in spontaneous and induced labor [24]. Unlike nulliparity, this is a modifiable risk factor that can be prevented by using different labor management. The use of a check-list to prevent and treat tachysystole, as suggested by some authors [25], could be a helpful tool. Interestingly, in our series, tachysystole was not linked to FHR ACOG Category IIIII in 22 cases (42\%), but it still resulted in asphyxia.

From the review of FHR tracing prospectively collected and managed according to ACOG algorithms, we observed that FHR Category II or III occurred in $50.5 \%$ of asphyxiated neonates. It preceded HIE in $56.3 \%$ of cases.

As recently reported [14], we acknowledge the limitations of electronic FHR monitoring in predicting perinatal asphyxia and the poor specificity of this test. Interestingly, our findings are similar to those of Clark, who using the same classification, observed that of infants who are born with metabolic acidemia, only approximately one-half could be identified with FHR monitoring.

Independently from the efforts at training and at applying uniform algorithms during labor, it may be unrealistic to expect high sensitivities for the detection of asphyxia using FHR alone. This underscores thefact that key management decisions in preventing asphyxia should not be based only on FHR interpretation. Other factors, including maternal and fetal history, stage and progress of labor and signs of fetal distress in labor, should be taken into account. Some Authors have suggested that a physiological interpretation of the FHR recordings may improve our understanding offetal oxygenation during labor $[26,27]$. Whether such interpretation may prevent asphyxia is unproven.

Our study has some limitations. First of all, the number of cases included in the study is not large. In addition, some cases were excluded due to lack of information or refusal of consent. The strengths of our study are that umbilical cord gas analysis was done consistently across the participating institutions; and the prospective nature of the study, with hospitals using shared protocols of maternal and neonatal care, as well as of methods of induction, labor management, oxytocin use, and FHR tracing interpretation.

Furthermore, all FHR tracings were reviewed in order to standardize their interpretations. In addition, for every neonate we have the values of both $\mathrm{pH}$ and $\mathrm{BE}$ and lactate, thus permitting to have a more realistic picture of all nuances of acid-base status, and not only the most extreme. In our network, all cases of HIE were promptly identified with a limited number of newborns transferred. Shared care protocols and cooperative management system has guaranteed appropriate care for all asphyxiated neonates. We hope that long-term neurodevelopmental follow up data will help us to better define risk factors for neurological sequelae in asphyxiated neonates, allowing the development of adequate rehabilitation programs for these children.

\section{Conclusions}

We identified at least one risk factor associated with all cases of HIE and with the majority of cases of perinatal asphyxia. In the absence of risk factors, the probability of developing perinatal asphyxia resulted extremely low. Among risk factors, the most frequent were nulliparity and tachysystole. Sentinel events confirmed their significant role as antecedents of asphyxia. Our findings on FHR tracing support that it is unrealistic to expect high sensitivities for the detection of asphyxia using FHR alone. FHR interpretation should consider the background risk factors. Future studies will address the question of whether a more integrated approach taking into account risk factors and new adjunctive monitoring methods can improve awareness of fetal status and, consequently, prediction of asphyxia.

\section{Abbreviations}

HIE: Hypoxic-ischemic encephalopathy; FHR: Fetal heart rate; BE: Base Excess; UA: Umbilical Artery; ACOG: American College of Obstetricians and Gynecologists; TS: Thomson score; CS: Caesarean Section; GA: Gestational Age; VD: Vaginal Delivery; OVD: Operative Vaginal Delivery

\section{Acknowledgements}

Not applicable

\section{Authors' contributions}

AL: study design, data analysis, FHR tracing revision, manuscript preparation and final review of the manuscript. LL: study design, data collection and analysis, FHR tracing revision, manuscript preparation. Ml: study design, data collection, FHR tracing revision and final review of the manuscript. FB: study design, data collection and FHR tracing revision. MF: study design, data collection and FHR tracing revision. SM: study design, data collection and neonatal follow-up. FT: study design, data collection and neonatal follow-up. PC: study design, data collection and neonatal follow-up. TV: study design, data collection and neonatal follow-up. GP: study design, data analysis, neonatal follow-up, manuscript preparation and final review of the manuscript. All authors read and approved the final manuscript.

\section{Funding}

The study was financed by University of Milano Bicocca (14291 2015-ATE0324 ATE - Fondo di Ateneo 2015).

\section{Availability of data and materials}

Data on individual patient are available upon request.

\section{Ethics approval and consent to participate}

The study was approved by the Institutional Review Board (San Gerardo Hospital's Ethical Committee, 23rd December 2014, reference number 482, informed consent included). Written informed consent was obtained from all participants.

\section{Consent for publication}

Not applicable

Competing interests

The authors declare that they have no competing interests. 


\section{Author details}

'Department of Obstetrics and Gynecology, ASST Vimercate, Carate B.za Hospital, University of Milano-Bicocca, Monza, Italy. ${ }^{2}$ Department of Obstetrics and Gynecology, Fondazione MBBM, San Gerardo Hospital, University of Milano-Bicocca, Monza, Italy. ${ }^{3}$ Department of Obstetrics and Gynecology, ASST Vimercate, Vimercate Hospital, Vimercate, Italy. ${ }^{4}$ Neonatal Intensive Care Unit, Fondazione MBBM, San Gerardo Hospital, Monza, Italy. ${ }^{5}$ Department of Pediatrics, ASST Vimercate, Carate B.za Hospital, Vimercate, Italy. ${ }^{6}$ Department of Pediatrics, ASST Vimercate, Vimercate Hospital, Vimercate, Italy. ${ }^{7}$ Department of Pediatrics, ASST Monza, Desio Hospital, Desio, Italy.

Received: 11 September 2019 Accepted: 16 March 2020 Published online: 30 March 2020

\section{References}

1. American College of Obstetricians and Gynecologists. "Neonatal encephalopathy and neurologic outcome." The American College of Obstetricians and Gynecologists, American Academy of Pediatrics. 2nd ed. 2014.

2. Graham EM, Ruis KA, Hartman AL, Northington FJ, Fox HE. A systematic review of the role of intrapartum hypoxia-ischemia in the causation of neonatal encephalopathy. Am J Obstet Gynecol. 2008;199(6):587-95.

3. Sarnat HB, Sarnat MS. Neonatal encephalopathy following fetal distress. A clinical and electroencephalographic study. Arch Neurol. 1976;33(10): 696-705.

4. Thompson CM, Puterman AS, Linley LL, et al. The value of a scoring system for hypoxic ischaemic encephalopathy in predicting neurodevelopmental outcome. Acta Paediatr. 1997;86(7):757-61.

5. Malin GL, Morris RK, Khan KS. Strength of association between umbilical cord $\mathrm{pH}$ and perinatal and long term outcomes: systematic review and meta-analysis. BMJ. 2010;340:C1471.

6. Kelly $\mathrm{R}$, Ramaiah $\mathrm{S}$, Sheridan $\mathrm{H}$, et al Dose-dependent relationship between acidosis at birth and likelihood of death or cerebral palsy archives of disease in childhood - fetal and neonatal edition published online first: 082017.

7. Okereafor A, Allsop J, Counsell S, et al. Patterns of brain injury in neonates exposed to perinatal sentinel events. Pediatrics. 2008;121(5):906-14.

8. Badawi N, Kurinczuk JJ, Keogh JM, et al. Antepartum risk factors for newborn encephalopathy: the Western Australian case-control study. BMJ. 1998;317(7172):1549-53.

9. Badawi N, Kurinczuk JJ, Keogh JM, et al. Intrapartum risk factors for newborn encephalopathy: the Western Australian case-control study. BMJ. 1998; 317(7172):1554-8.

10. Locatelli A, Incerti M, Paterlini G, et al. Antepartum and intrapartum risk factors for neonatal encephalopathy at term. Am J Perinatol. 2010;27(8): 649-54

11. Hayes BC, McGarvey C, Mulvany S, et al. A case-control study of hypoxicischemic encephalopathy in newborn infants at $>36$ weeks gestation. Am J Obstet Gynecol. 2013;209(1):29.e1-29.e19.

12. Liljestrom L, Wikstrom AK, Agren J, Jonsson M. Antepartum risk factors for moderate to severe neonatal hypoxic ischemic encephalopathy: a Swedish national cohort study. Acta Obstet Gynecol Scand. 2018;97(5):615-23.

13. Nelson KB, Bingham P, Edwards EM, et al. Antecedents of neonatal encephalopathy in the Vermont Oxford network encephalopathy registry. Pediatrics. 2012;130(5):878-86.

14. Clark SL, Hamilton EF, Garite TJ, Timmins A, Warrick PA, Smith S. The limits of electronic fetal heart rate monitoring in the prevention of neonatal metabolic acidemia. Am J Obstet Gynecol. 2017;216(2):163.e1-6.

15. Nelson KB, Sartwelle TP, Rouse DJ. Electronic fetal monitoring, cerebral palsy, and caesarean section: assumptions versus evidence. BMJ. 2016; 355:16405.

16. Società Italiana di Neonatologia. Raccomandazioni per l'assistenza al neonato con encefalopatia ipossico ischemica candidato al trattamento ipotermico. 2012. Biomedia SRL) http://backoffice.neonatologia.it/upload/1 994_RACCOMANDAZIONI_ASSISTENZA_NEONATO.pdf.

17. Clark SL, Nageotte MP, Garite TJ, et al. Intrapartum management of category II fetal heart rate tracings: towards standardization of care. Am J Obstet Gynecol. 2013;209(2):89-97.

18. Kurinczuk JJ, White-Koning M, Badawi N. Epidemiology of neonatal encephalopathy and hypoxic-ischaemic encephalopathy. Early Hum Dev. 2010;86(6):329-38
19. Volpe JJ. Hypoxic-ischemic encephalopathy: biochemical and physiological aspects. In: Volpe JJ, editor. Neurology of the newborn. Philadelphia: Saunders; 2001. p. 217-76

20. Jonsson M, Agren J, Norden-Lindeberg S, Ohlin A, Hanson U. Neonatal encephalopathy and the association to asphyxia in labor. Am J Obstet Gynecol. 2014;211(6):667.e1-8.

21. Nelson DB, Lucke AM, McIntire DD, Sanchez PJ, Leveno KJ, Chalak LF. Obstetric antecedents to body-cooling treatment of the newborn infant Am J Obstet Gynecol. 2014;211:155.e1-6.

22. Lundgren C, Brudin L, Wanby AS, Blomberg M. Ante-and intrapartum risk factors for neonatal hypoxic ischemic encephalopathy. J Matern Fetal Neonatal Med. 2018;31:1595-601

23. Liljestrom L, Wikstrom AK, Jonsson M. Obstetric emergencies as antecedents to neonatal hypoxic ischemic encephalopathy, does parity matter? Acta Obstet Gynecol Scand. 2018:97:1396-404.

24. Heuser CC, Knight S, Esplin MS, et al. Tachysystole in term labor: incidence, risk factors, outcomes, and effect on fetal heart tracings. Am J Obstet Gynecol. 2013;209:32.e1-6.

25. Clark S, Belfort M, Saade G, et al. Implementation of a conservative checklistbased protocol for oxytocin administration: maternal and newborn outcomes. Am J Obstet Gynecol. 2007;197:480.e1-5.

26. Ugwumadu A. Are we (mis)guided by current guidelines on intrapartum fetal heart rate monitoring? Case for a more physiological approach to interpretation. BJOG. 2014:121:1063-70.

27. Pinas A, Chandraharan E. Continuous cardiotocography during labour analysis, classification and management. Best Pract Res Clin Obstet Gynaecol. 2016;30:33-47.

\section{Publisher's Note}

Springer Nature remains neutral with regard to jurisdictional claims in published maps and institutional affiliations.

Ready to submit your research? Choose BMC and benefit from:

- fast, convenient online submission

- thorough peer review by experienced researchers in your field

- rapid publication on acceptance

- support for research data, including large and complex data types

- gold Open Access which fosters wider collaboration and increased citations

- maximum visibility for your research: over $100 \mathrm{M}$ website views per year

At $\mathrm{BMC}$, research is always in progress.

Learn more biomedcentral.com/submission 